\title{
Algorithm and Accuracy Analysis of Weighted Maximum Likelihood Estimation in Multi-station DF Crossing Localization Zong Junjun ${ }^{1, a^{*}}$,Cui Xunxue ${ }^{2, b}$,Yang Hui ${ }^{3, c}$, Gao Bin ${ }^{4, d}$ \\ 1,2,3,4 New Star Research Institute of Applied Technology, Hefei, 230031,P.R.China \\ azjj_2008@163.com, ${ }^{\text {b }}$ cuixx_2013@163.com, 'sanpedroman@163.com, ${ }^{\text {d5 } 563321248 @ q q . c o m ~}$
}

Corresponding Author: Zong Junjun.Tel:18909699781. E-mail:zjj_2008@163.com

Keywords: passive localization; direction-finding (DF); crossing localization; weighted maximum likelihood estimation (WMLE); error analysis

\begin{abstract}
In order to solve the non uniform environment of the target area influencing the variance of DF error, the weighted maximum likelihood estimation (WMLE) algorithm was proposed. In this algorithm, the effect of the target distance was introduced into MLE. we construct the weighted vector to make up for the effect when the target distance increase the variance of the DF error become worse. Theoretical analysis showed that the algorithm of WMLE could further improve the accuracy of the multi-station DF crossing localization.
\end{abstract}

Passive acoustic localization has received good attention in the world and obtained considerable development in the military fields, for its good concealment, strong confidentiality and less susceptible to interference. Among its many location methods, multi-station DF crossing location is one of the important. It is a kind of method, by using multi-station DF information to obtain the location of target, also known as triangulation method. The location accuracy is mainly affected by the DF's accuracy, array's quantity, base-station's configuration, localization's algorithm and other factors, especially when the sensor's device and array's configuration was constrained, location algorithm would become the main factors affecting the location accuracy.

Recently years, many mature localization algorithms have been formed, such as least squares estimation (LSE), pseudo-linear estimation (PLE), maximum likelihood estimation (MLE). Among them, the performance of MLE is excellent. When using the MLE to locate the sound point of the burst, we usually assume the variance ( $\sigma$ ) of DF error is unchanged. But actually, target region isn't an uniform environment, $\sigma$ will be influenced by the target distance. Obviously, from the control of estimation error, MLE isn't an effective method. This article according to the point of the account source, based on the view of Dogancay's MLE, we construct the WMLE, namely, by setting the weighted function to compensate for the effect of heterogeneous environment on DF error.

\section{Location Principle}

In figure 1, The station position is represented by $S_{k}=\left(x_{k}, y_{k}, z_{k}\right)^{T}(k=1,2, \cdots, n)$, where

$T$ denotes matrix transpose. The true location of the stationary target is represented by 
$X_{P}=\left(x_{p}, y_{p}, z_{p}\right)^{T}$. We collect azimuth $\left(\theta_{k}\right)$ and elevation $\left(\phi_{k}\right)$ angle at each station. The unit of measurement is radian. It is assumed that azimuth and elevation angles are independent of each other, which satisfied with zero mean Gaussian noise, and their mean-square-deviation are denoted as $\sigma_{\theta_{k}}$ and $\sigma_{\phi_{k}}$.

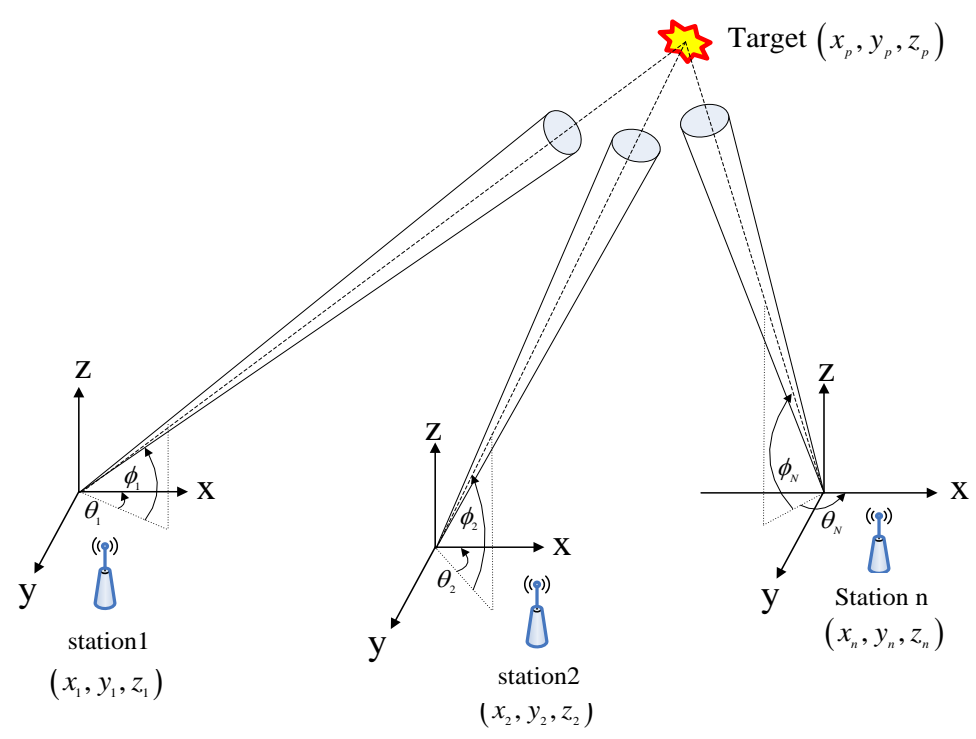

Figure.1. 3-D AOA based source localization for noisy case

According to the relations between stations and emitter sources, we can get equation (1) and (2)

$$
\begin{aligned}
& \theta_{k}=\tan ^{-1}\left(\frac{y-y_{k}}{x-x_{k}}\right) \\
& \phi_{k}=\tan ^{-1}\left(\frac{z_{p}-z_{k}}{\sqrt{\left(x_{p}-x_{k}\right)^{2}+\left(y_{p}-y_{k}\right)^{2}}}\right)
\end{aligned}
$$

\section{Weighted Maximum Likelihood Estimation}

According to the principle of maximum likelihood estimation, we can construct the maximum likelihood cost function

$$
J_{M L}(p)=e^{T}(p) W^{-1} e(p)
$$

Where $W$ is a covariance matrix of the bearing noise and $e(p)$ is the error vector, which can be expressed as

$$
\begin{aligned}
& W=\operatorname{diag}\left\{\sigma_{\theta_{1}}^{2}, \sigma_{\theta_{2}}^{2}, \cdots, \sigma_{\theta_{n}}^{2}, \sigma_{\phi_{1}}^{2}, \sigma_{\phi_{2}}^{2}, \cdots, \sigma_{\phi_{n}}^{2}\right\} \\
& e(p)=\left[\tilde{\theta}_{1}-\theta_{1}(p), \cdots, \tilde{\theta}_{n}-\theta_{n}(p), \tilde{\phi}_{1}-\phi_{1}(p), \cdots, \tilde{\phi}_{n}-\phi_{n}(p)\right]
\end{aligned}
$$

Therefore, the estimation value of the emitter source can be expressed as

$$
\hat{p}_{M L}=\underset{p}{\arg \min } J_{M L}(p)
$$

The maximum likelihood location estimator is an nonlinear equation, so it does not have a closed-form solution and requires the use of a numerical search algorithm. The Gauss-Newton (GN) 
algorithm, which is a batch iterative minimization technique, is often employed to calculate the MLE. The GN algorithm consists of

$$
\mathbf{X}_{i+1}=\mathbf{X}_{i}-\left(\mathbf{J}_{i}^{T} \mathbf{W}^{-1} \mathbf{J}_{i}\right)^{-1} \mathbf{J}_{i}^{T} \mathbf{W}^{-1} \mathbf{e}\left(\mathbf{P}_{i}\right)
$$

Where $J_{i}$ is the $2 \mathrm{~N} \times 3$ Jacobian of $e(p)$

$$
\mathbf{J}_{i}=\left[\begin{array}{ccc}
\frac{\sin \hat{\phi}_{1}(i)}{\hat{d}_{i 1}} & \frac{-\cos \hat{\phi}_{1}(i)}{\hat{d}_{i 1}} & 0 \\
\vdots & \vdots & \vdots \\
\frac{\sin \hat{\phi}_{n}(i)}{\hat{d}_{i n}} & \frac{-\cos \hat{\phi}_{n}(i)}{\hat{d}_{i n}} & 0 \\
\frac{\sin \hat{\theta}_{1}(i) \cos \hat{\phi}_{1}(i)}{\left\|\mathbf{X}_{i}-\mathbf{S}_{i}\right\|} & \frac{\sin \hat{\theta}_{1}(i) \sin \hat{\phi}_{1}(i)}{\left\|\mathbf{X}_{i}-\mathbf{S}_{i}\right\|} & \frac{-\cos ^{2} \hat{\theta}_{1}(i)}{\hat{d}_{i 1}} \\
\vdots & \vdots & \vdots \\
\frac{\sin \hat{\theta}_{n}(i) \cos \hat{\phi}_{n}(i)}{\left\|\mathbf{X}_{i}-\mathbf{S}_{n}\right\|} & \frac{\sin \hat{\theta}_{n}(i) \sin \hat{\phi}_{n}(i)}{\left\|\mathbf{X}_{i}-\mathbf{S}_{n}\right\|} & \frac{-\cos ^{2} \hat{\theta}_{n}(i)}{\hat{d}_{i n}}
\end{array}\right]
$$

Where $\hat{d}_{i k}=\left\|\mathbf{X}_{i}(1: 2)-\mathbf{S}_{k}(1: 2)\right\|$,denote as the distance between the $i$ th emitter source and the $k$ th station. $\mathbf{X}_{i}(1: 2) 、 \mathbf{S}_{k}(1: 2)$ is a $2 \times 1$ vector containing the first two entries of $X_{i}$. The initial coordinate of the target can be calculated by the PLE.

From the above GN iterative, we can find the variance of DF error of azimuth $\sigma_{\varphi}$ and elevation $\sigma_{\theta}$ is unchanging, but actually, DF error is influenced by the target distance, usually it will become big with the increase of the distance. Here, we assume that the variance of DF error of azimuth and elevation is $\sigma$ when the distance is $\mathbf{d}$, So we can draw $\sigma_{i}$ corresponding to the target distance $d_{i}$. The relationship can be expressed as

$$
\begin{aligned}
& \sigma_{\varphi_{k i}}=\frac{d_{k i}}{\mathbf{d}} \sigma_{\varphi_{k}} \\
& \sigma_{\theta_{k i}}=\frac{d_{k i}}{\mathbf{d}} \sigma_{\theta_{k}}
\end{aligned}
$$

The covariance matrix (W)of the DF error can be expressed as

$$
\mathbf{W}_{i}=\operatorname{diag}\left\{\sigma_{\theta_{1 i}}^{2}, \sigma_{\theta_{2 i}}^{2}, \cdots, \sigma_{\theta_{n i}}^{2}, \sigma_{\phi_{1 i}}^{2}, \sigma_{\phi_{2 i}}^{2}, \cdots, \sigma_{\phi_{n i}}^{2}\right\}
$$

\section{Simulation Experiment}

Experiment Condition. Assuming the system is conformed by six base stations, which has uniform linear array and located at the $x$ axis from -3000 to 3000 . And the area of target is assumed as: axis $x$ is $\pm 3 \mathrm{~km}$, axis $y$ is $1 \mathrm{~km} \sim 7 \mathrm{~km}$,axis $z$ is $\pm 0.5 \mathrm{~km}$. The variance $\sigma$ of azimuth and elevation is assumed as $0.5^{0} 、 1.0^{0} 、 1.5^{0} 、 2.0^{0} 、 2.5^{0} 、 3.0^{0}$.

Experiment result and Result analysis. Figure 2, figure 3 and Figure 4 are RMSE's curve of 1000 Monte-Carlo experiment results of PLE, MLE, WMLE and CRLB. The unit of $x$ axis is degree, $y$ axis is meter. In figure 2, the variance of azimuth $\sigma_{\varphi}$ and elevation $\sigma_{\theta}$ are equal, they changed from $0.5^{0}$ to $3^{0}$ at the same time. In figure $3, \sigma_{\varphi}=0.5^{0}, \sigma_{\theta}$ changed from $0.5^{0}$ to $3^{0}$.In figure $4, \sigma_{\theta}=0.5^{0}, \sigma_{\varphi}$ changed from $0.5^{0}$ to $3^{0}$. 


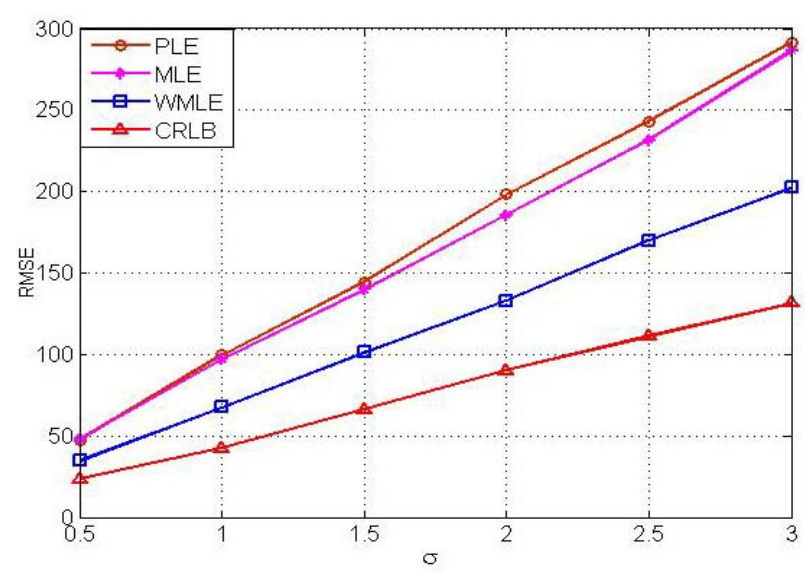

Figure.2. Performance comparison of RMSE for $\sigma_{\varphi}=\sigma_{\theta}=\sigma$

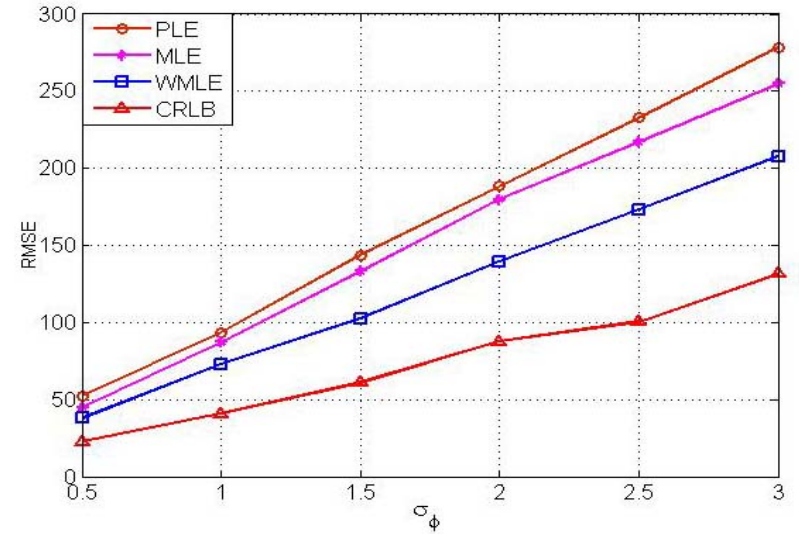

Figure.3. Performance comparison of RMSE for $\sigma_{\theta}=0.5^{0}$

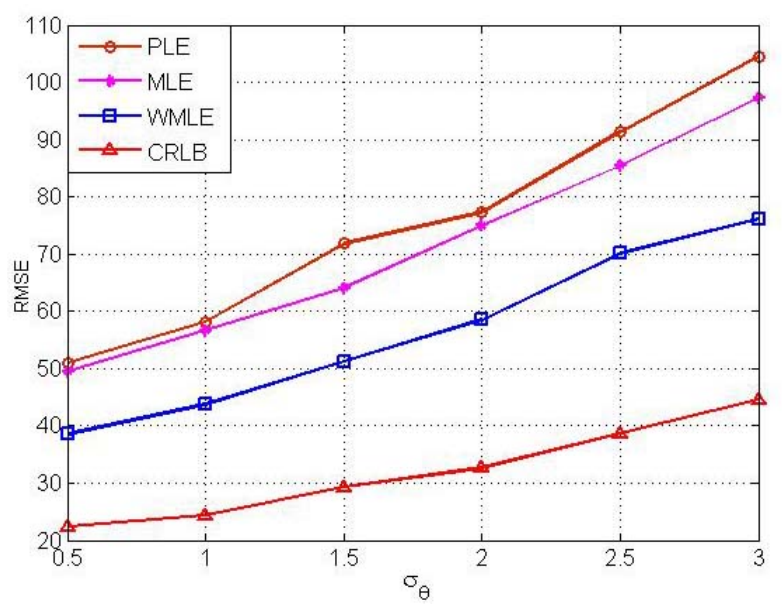

Figure.4. Performance comparison of RMSE for $\sigma_{\varphi}=0.5^{0}$

From the curves of the figure, we can draw a conclusion:

(1)The location accuracy of the three algorithms will reduce with the variance of azimuth $\sigma_{\varphi}$ and elevation $\sigma_{\theta}$ increasing. Among them, the accuracy of PLE is the worst, the accuracy of WMLE is the best, for the curve of WMLE is approach to CRLB. And the accuracy of MLE is between PLE and WMLE, for the curve is at the middle of them. So if we don't change the other condition of the system and only transform algorithm, the WMLE can further improve the location accuracy of the system.

(2) The variance of azimuth $\sigma_{\varphi}$ and elevation $\sigma_{\theta}$,when one fixed the other changing, conclusion (1) still holds, but comparing figure 3 and figure 4 we can find, RMSE was influenced higher by 
$\sigma_{\varphi}$. Namely the location accuracy is influenced significantly higher by elevation DF error than azimuth DF error. Therefore, in condition of 3D, improving the accuracy of elevation angle is more helpful for multi-station DF crossing Location.

\section{Conclusion}

This paper studied the algorithm of the multi-station DF crossing localization. based on the MLE, the WMLE algorithm was proposed which considering the influence of the target distance.

Experiment results show that the location accuracy of WMLE is much higher than that of PLE. which can make up of the error of the fixed variance and improve the influence of nonhomogeneous environment of the target area, compared with the MLE algorithm, WMLE can further improve the accuracy of the multi-station DF crossing localization.

\section{Acknowledgement}

This work was financially supported by the Fund of National Natural Science(61170252).

\section{References}

[1] Bishop,A.A.,Pathirana,P.N., A discussion on passive location discovery in emitter networks Using angle-only measurements. In proceedings of the 2006 International Wireless Communications and Mobile Computing Conference, Vancouver, BC,Canada(2006),p.1337

[3] Kostas E B,Max G,Lydia E K. Evaluation of Algorithms for Bearing-only SLAM[C]//Robotics and Automation(2006). ICRA 2006. in Proceedings of IEEE International Conference, Vol. 15-19 ( 2006), p.1937

[4] K.Dogancay,”3D passive localization in the presence of large bearing noise," in Proc.13th European Signal Processing Conference, EUSIPCO 2005, Antalya, Turkey, September 2005, in Press.

[5] H.Hmam,K.Dogancay,"Passive localization of scanning emitters," in proceeding of IEEE Transactions on aerospace and electronic systems, vol.46,no.2,pp.944-951,April 2010.

[6] R.Badeau, B.David.'Weighted maximum likelihood autoregressive and moving average spectrum modeling," in Proceeding of IEEE International Conference on Acoustics, Speech and Signal Processing(ICSAAP),2008,pp.3761-3764. 